\title{
Analysis of the Dynamics of Particle Swarm Optimization
}

\author{
Keiichiro Yasuda* Member \\ Nobuhiro Iwasaki ${ }^{*}$ Student Member
}

This paper deals with the stability analysis of PSO in order to obtain an understanding about how it searches a globally optimal solution and strategies about how to tune its parameters. While two types of generalized reduced models of PSO are proposed to analyze the dynamics of PSO, the stability analysis is carried out on the basis of both the eigenvalue analysis and the bounded input bounded output stability.

Keywords: Meta-heuristics, Global Optimization, Particle Swarm Optimization, Stability Analysis

\section{Introduction}

The Particle Swarm Optimization (PSO) method is one of the most powerful methods for solving unconstrained and constrained global optimization problems. The method was originally proposed by J. Kennedy as an optimization method in $1995^{(1)}$ and has been proved to be an efficient method for many global optimization problems. Little is, however, known about how the PSO method works or finds a globally optimal solution of a problem.

This paper deals with the analysis of the dynamics of PSO in order to obtain an understanding about how it searches a globally optimal solution and strategies about how to tune its parameters. While two types of generalized reduced models of PSO are proposed in order to analyze the dynamics of PSO, the stability analysis is carried out on the basis of both the eigenvalue analysis and Bounded Input Bounded Output (BIBO) stability.

\section{Particle Swarm Optimization Algorithm}

Each particle has its own position, $x$, transfer vector, $v$, and own best position visited thus far, pbest. Furthermore, all of the particles share the best position visited by all of the particles thus far, gbest. A particle forms a new transfer vector by linearly combining three vectors. In the $(k+1)$-th transfer, the $j$-th coordinate component of the transfer vector of the $i$-th particle is manipulated according to the following equation:

$$
\begin{aligned}
v_{i j}^{k+1}= & w \cdot v_{i j}^{k}+c_{1} \cdot \operatorname{rand}_{1}() \cdot\left(\text { pbest }_{i j}^{k}-x_{i j}^{k}\right) \\
& +c_{2} \cdot \operatorname{rand}_{2}() \cdot\left(\text { gbest }_{j}^{k}-x_{i j}^{k}\right) \cdots \cdots
\end{aligned}
$$

where $i=1, \cdots, m$ ( $m$ is the size of the swarm), $j=1, \cdots, n$ ( $n$ is the size of space of a given problem), $w, c_{1}$ and $c_{2}$ are positive constants, $\operatorname{rand}_{1}$ and $\operatorname{rand}_{2}$ are random numbers that are uniformly distributed in $[0,1]$, and $k$ determines the iteration number. Customarily, the transfer vector $v_{i j}$ is also referred to as the velocity in PSO, and the present paper follows this convention. Each particle moves according to the following equation:

$$
x_{i j}^{k+1}=x_{i j}^{k}+v_{i j}^{k+1} \ldots \ldots \ldots \ldots \ldots \ldots \ldots \ldots
$$

\footnotetext{
* Tokyo Metropolitan University

1-1, Minamiosawa, Hachioji-shi, Tokyo 192-0397
}

\section{Constant-p System of Particle Swarm Op-} timization

3.1 Constant-p System The above algorithm will be simplified in order to analyze the dynamics of the PSO algorithm. If the particle of the PSO algorithm is reduced to one dimension, then vector notation can be thrown out and the particle's trajectory can be displayed as a simple graph. Without any information, the two terms of the formula can be transformed into one term $^{(1)}$ :

$$
\begin{aligned}
v^{k+1} & =w \cdot v^{k}+\phi \cdot\left(p^{k}-x^{k}\right) \ldots \ldots \ldots \ldots \ldots \ldots(3) \\
\phi & =c_{1} \cdot \operatorname{rand}_{1}()+c_{2} \cdot \operatorname{rand}_{2}() \cdots \ldots \ldots \ldots(4) \\
p^{k} & =\frac{c_{1} \cdot \operatorname{rand}_{1}() \cdot \operatorname{pbest}^{k}+c_{2} \cdot \operatorname{rand}_{2}() \cdot \text { gbest }^{k}}{c_{1} \cdot \operatorname{rand}_{1}()+c_{2} \cdot \operatorname{rand}_{2}()}
\end{aligned}
$$

where $p$ is the weighted average of the two best, and $\phi$ is a random number distributed in $\left[0,\left(c_{1}+c_{2}\right)\right]$ and its average value is $\frac{c_{1}+c_{2}}{2}$.

The trajectory of the particle can be plotted and studied when the weighted best point $p$ and the random number $\phi$ are made constant $\phi=\frac{c_{1}+c_{2}}{2}$. Therefore there is no randomness and no vector notation is necessary. Keep in mind that the simplified model does not represent the original PSO situation. In general population sizes are greater than one and seldom a particle operates on only one dimension.

The constant- $p$ system of PSO can be expressed as follows:

$$
\left\{\begin{array}{l}
v^{k+1}=w \cdot v^{k}+\phi \cdot y^{k} \\
y^{k+1}=-w \cdot v^{k}+(1-\phi) \cdot y^{k}
\end{array}\right.
$$

where $y^{k}=p-x^{k}$.

3.2 Stability Analysis of Constant-p System The constant- $p$ system can be written in terms of matrix algebra.

$$
\left[\begin{array}{l}
v^{k+1} \\
y^{k+1}
\end{array}\right]=\left[\begin{array}{cc}
\omega & \phi \\
-\omega & 1-\phi
\end{array}\right]\left[\begin{array}{l}
v^{k} \\
y^{k}
\end{array}\right] \stackrel{\text { def }}{=} M\left[\begin{array}{l}
v^{k} \\
y^{k}
\end{array}\right]
$$$$
\text { ................. (7) }
$$

where $M$ is the matrix of the system.

The dynamics of the constant- $p$ system is completely 
defined by $M$. According to the stability theory, the behavior of the particle is asymptotically stable if and only if $\left|\lambda_{1}\right|<1$ and $\left|\lambda_{2}\right|<1$, where $\lambda_{1}$ and $\lambda_{2}$ are the eigenvalues of the matrix $M$. By using the parameters $\phi$ and $w$, the criterion of convergence $\left(\left|\lambda_{1}\right|<1\right.$ and $\left.\left|\lambda_{2}\right|<1\right)$ can be written as follows:

$$
\begin{aligned}
& 0<\phi<2 w+2 \ldots \ldots \ldots \ldots \ldots \ldots \ldots \ldots \ldots \ldots \ldots \ldots \ldots \ldots \ldots \ldots \ldots \ldots \ldots \ldots \ldots \ldots \\
& 0 \leq w<1
\end{aligned}
$$

Since $|\lambda|=\sqrt{w}$ in case where $w+1-2 \sqrt{w}<\phi<$ $w+1+2 \sqrt{w}$ (i.e., $\left.(1-\sqrt{w})^{2}<\phi<(1+\sqrt{w})^{2}\right)$, the stability of the constant- $p$ system is completely defined by $w$. The stability of each particle, however, can be exactly evaluated based on this model when the fluctuation of $p$ is bounded. Since the search space (feasible region) is generally limited in global optimization problems, the fluctuation of $p$ is also bounded.

\section{Varying- $p$ System of Particle Swarm Opti- mization}

4.1 Varying- $\boldsymbol{p}$ System The weighted best point $p$ is not static but dynamic, often exhibiting complex behavior. Therefore the constant- $p$ system is contrived only to provide some information on how each particle behaves without the interaction among the particles. The following system, named a varying- $p$ system, where $p$ is picked up is proposed in order to consider the effect of $p$ in this section ${ }^{(2)}$.

$$
\begin{aligned}
{\left[\begin{array}{l}
v^{k+1} \\
x^{k+1}
\end{array}\right] } & =\left[\begin{array}{cc}
\omega & -\phi \\
\omega & 1-\phi
\end{array}\right]\left[\begin{array}{l}
v^{k} \\
x^{k}
\end{array}\right]+\left[\begin{array}{l}
\phi \\
\phi
\end{array}\right] p^{k} \\
& \stackrel{\text { def }}{=} M^{\prime} \quad\left[\begin{array}{l}
v^{k} \\
x^{k}
\end{array}\right]+N \begin{array}{ll}
N & p^{k}
\end{array}
\end{aligned}
$$

This system is a linear time-invariant system with input $p$ which is the weighted average of the two best (pbest and gbest). It is clear that the eigenvalues of the matrix $M$ in Equation (7) correspond to the eigenvalues of the matrix $M^{\prime}$ in Equation (10).

4.2 Stability Analysis of Varying- $p$ System The input $p^{k}$, which is the weighted average of the two best (pbest and gbest) is also bounded when the search space of a given problem is bounded. Since the search space (feasible region) is generally limited in global optimization problems, the stability of the above varying- $p$ system can be evaluated by Bounded Input Bounded Output (BIBO) stability. It is well known that BIBO stability implies asymptotic stability.

Suppose that the input $p^{k}$ is bounded, so that

$$
\left\|p^{k}\right\| \leq C_{1}<\infty \ldots \ldots \ldots \ldots \ldots \ldots \ldots \ldots
$$

Then the outputs $v$ and $x$ are also bounded if $\left|\lambda_{1}^{\prime}\right|<1$ and $\left|\lambda_{2}^{\prime}\right|<1$, where $\lambda_{1}^{\prime}$ and $\lambda_{2}^{\prime}$ are the eigenvalues of the matrix $M^{\prime}$.

$$
\begin{aligned}
\left\|v^{k+1}\right\| & \leq C_{2}<\infty \ldots \ldots \ldots \ldots \ldots \ldots \ldots \ldots \ldots \\
\left\|x^{k+1}\right\| & \leq C_{3}<\infty \ldots \ldots \ldots \ldots \ldots \ldots \ldots \ldots
\end{aligned}
$$

Since the random number $\phi$ is made constant in the above varying- $p$ system, $p^{k}$ will correspond to the best point of the swarm gbest when $c_{1}=0, c_{2}=1$. In

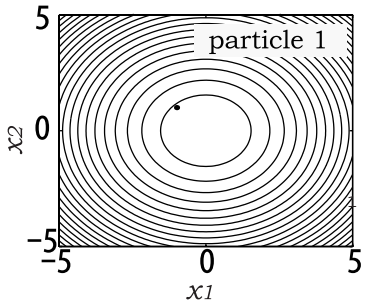

Convex Function $f_{1}$

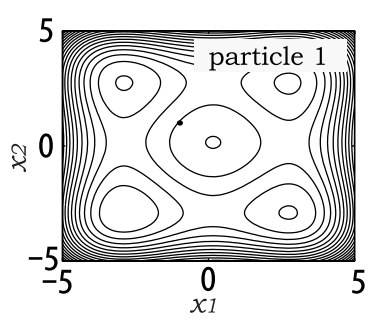

Nonconvex Function $f_{2}$
Fig. 1. Points searched by a particle of original PSO for objective functions $f_{1}$ and $f_{2}$

the PSO algorithm, $p^{k}$ is not only the input but also the asymptotically stable equilibrium point of the deterministic PSO system expressed in Equation (10).

On the other hand, the transition of the best point $p^{k}$ searched by the swarm is strongly dependent on the shape of the given objective function.

\section{A Simple Simulation Result}

The results of applying the original PSO with 20 particles to two types of objective functions (i.e., a convex function $f_{1}$ and a nonconvex function $f_{2}$ ) are shown in Fig.1. Points, which are searched by particle 1 of the swarm consisting of 20 particles, are plotted for $f_{1}$ and $f_{2}$, respectively.

From Fig.1, it is clear that the transition of best points searched by each particle reflects the shape of the objective functions $f_{1}$ and $f_{2}$, i.e., while intensive search is realized for the convex objective function $f_{1}$, diverse search is realized for the nonconvex objective function $f_{2}$. In other words, it is numerically confirmed that PSO has an autonomous mechanism that reflects the shape of an objective function so as to coordinate the intensification and the diversification of search process.

\section{Conclusion}

In this paper, two different types of reduced systems (i.e., constant- $p$ system and the varying- $p$ system) are proposed in order to analyze the behavior of PSO.

Based on the numerical simulations using typical convex and nonconvex objective functions and the stability analysis of the constant- $p$ system and the varying- $p$ system, it is estimated that PSO has the autonomous mechanism which reflects the shape of an objective function to be optimized.

Though the consideration of the random element of the PSO algorithm was disregarded in this paper, the consideration of the random element is one of the most important problems to be solved in the future.

This work was supported by Japan Society for the Promotion of Science (JSPS) - a Grant-in-Aid for Scientific Research (C) (15560252).

(Manuscript received Dec. 6, 2004)

\section{References}

(1) J. Kennedy and R. C. Eberhart: "Swarm Interlligence", Morgan Kaufmann Publishers (2001)

( 2 ) K. Yasuda, A. Ide, and N. Iwasaki: "Stability Analysis of Particle Swarm Optimization", Proceedings of The Fifth Metaheuristics International Conference, MIC03-34 (Aug. 2003) 